\title{
PROPUESTA CONCEPTUAL PARA LA MITIGACIÓN DE RIESGO POR INUNDACIÓN EN LA UNIDAD DE PLANEAMIENTO ZONAL (UPZ) TIBABUYES, LOCALIDAD DE SUBA
}

\section{CONCEPTUAL PROPOSAL FOR THE MITIGATION OF FLOODS RISKS IN THE ZONAL PLANNING UNIT TIBABUYES, SUBA LOCALITY}

\author{
${ }^{1}$ Tatiana Valbuena Villamil, ${ }^{2}$ Angie Lorena Ayala Rodríguez, \\ ${ }^{3}$ Didier Camilo Sierra Florez, ${ }^{4}$ Luz Adriana Gómez Rodríguez \\ ${ }^{1}$ Ingeniería Ambiental. Universidad Sergio Arboleda. Bogotá, Colombia \\ 2 PhD (C) en Ciencias Ambientales, Docente Facultad de Ciencias Exactas e ingeniería, \\ Universidad Sergio Arboleda. Bogotá, Colombia \\ ${ }^{3}$ Ingeniería Ambiental. Universidad Nacional Abierta y a Distancia. Bogotá, Colombia \\ ${ }^{1}$ Angie.ayala@usa.edu.co, ${ }^{2}$ Tatiana.valbuena@usa.edu.co, \\ ${ }^{3}$ Didier.sierra@usa.edu.co, ${ }^{4}$ Lagomezrodri@unadvirtual.edu.co
}

Citación: Valbuena, T., Ayala, A., Sierra, D., y Gómez, L. (2021). Propuesta conceptual para la mitigación de riesgo por inundación en la unidad de planeamiento zonal (UPZ) Tibabuyes, localidad de Suba. Revista de Investigación Agraria y Ambiental, 12(2), 199 - 214. DOI: https://doi.org/10.22490/21456453.3823

\section{RESUMEN}

Contextualización: la localidad de Suba, específicamente la Unidad de Planeamiento Zonal (UPZ) Tibabuyes, corresponde a un sector de la ciudad de Bogotá que, debido a su proceso de expansión desequilibrado, ha llevado a que las personas se asienten de manera ilegal sobre las Áreas Protegidas como el Humedal Juan Amarillo, el Humedal de la Conejera y la Zona de Manejo Especial del río Bogotá pertenecientes a la Estructura Ecológica Principal y se encuentren expuestos a fenómenos naturales como la inundación.

Vacío de conocimiento: Las incertidumbres en la variabilidad climática en zonas de vulnerabilidad hacen que la población sea propensa a sufrir accidentes de acuerdo a los posibles eventos naturales por una inadecuada planeación zonal y sobre todo una falta de planeación, es por esto que la investigación busca brindar diferentes herramienta metodológicas conceptuales para mejorar las condiciones que puedan llegar a afectar la calidad de vida de los habitantes y que puede llegar a generar consecuencias de ocupación de suelos importantes para el país y a aumentar significativamente el riesgo.

Propósito: diseñar una propuesta conceptual para reducir el riesgo de inundación en UPZ Tibabuyes.

Metodología: se usó un enfoque en la Acción Participativa (IAP) dentro de la cual se 
establecieron tres fases de investigación en donde se realizaron los análisis de percepción en la zona de acuerdo a las necesidades establecidas por la ronda hídrica, en la cual se pudo categorizar la amenaza según los escenarios de inundación; para esto, se determinaron los parámetros de evaluación (alta, cuando la lámina de agua es $>1,0 \mathrm{~m}$ y su velocidad $>$ $2,0 \mathrm{~m} / \mathrm{s}$ ) y la categoría (media, cuando la lámina de agua es $>0,5<1,0 \mathrm{~m}$ y su velocidad está entre 1,5 a 2,0 m/s).

Resultados y conclusiones: se determinó el plan de acción que consta de 19 estrategias, de las cuales 10 corresponden a estrategias implementadas es caso de presentarse un escenario de riesgo alto de inundación, y las 9 estrategias complementarias deben ser empleadas en caso de presentarse un escenario de riesgo medio de inundación. en ambas se relaciona la población afectada, los requerimientos y actividades técnicas por desarrollar junto con un presupuesto estimado y sus posibles fuentes de financiación.

Palabras clave: Amenaza; inundación; mitigación; riesgo; probabilidad

\section{ABSTRACT}

Contextualization: The locality of Suba, specifically the Tibabuyes Zonal Planning Unit (UPZ), corresponds to a sector of the city of Bogotá which, due to its unbalanced expansion process, has led people to settle illegally on Protected Areas such as the Juan Amarillo Wetland, the Conejera Wetland, and the Bogotá River Special Management Zone, which belong to the Main Ecological Structure and are exposed to natural phenomena such as flooding.
Knowledge Gap: the phenomenon of informal occupation has become a pattern that does not favor the sustainability of the city of Bogotá and affects the quality of life, poverty and a wide social problem that affects the inhabitants and that can generate important land occupation consequences for the country and significantly increase risk.

Purpose: to design a conceptual proposal to reduce the risk of flooding in UPZ Tibabuyes.

Methodology: a methodology focused on Participatory Action (PAR) was used, within which three research phases were established. where perception analyzes were carried out in the area according to the needs established by the water round, in which the threat could be categorized according to the flood scenarios, for this the evaluation parameters were determined (high, when the sheet of water is> $1.0 \mathrm{~m}$ and its speed is greater than $2.0 \mathrm{~m} / \mathrm{s}$ ), and the category (average, when the sheet of water is $>0.5<1.0 \mathrm{~m}$ and its speed is between 1.5 to $2.0 \mathrm{~m} / \mathrm{s}$ ).

Results and conclusions: The action plan consisting of 19 strategies was determined, out of which 10 correspond to implemented strategies in the event of a high flood risk scenario, and the 9 complementary strategies should be used in the event of a medium risk scenario. flood. In both, the affected population, the requirements and technical activities to be developed are related, together with an estimated budget and its possible sources of financing.

Keywords: Hazard; Flood; mitigation; risk, probability 


\section{RESUMEN GRÁFICO}

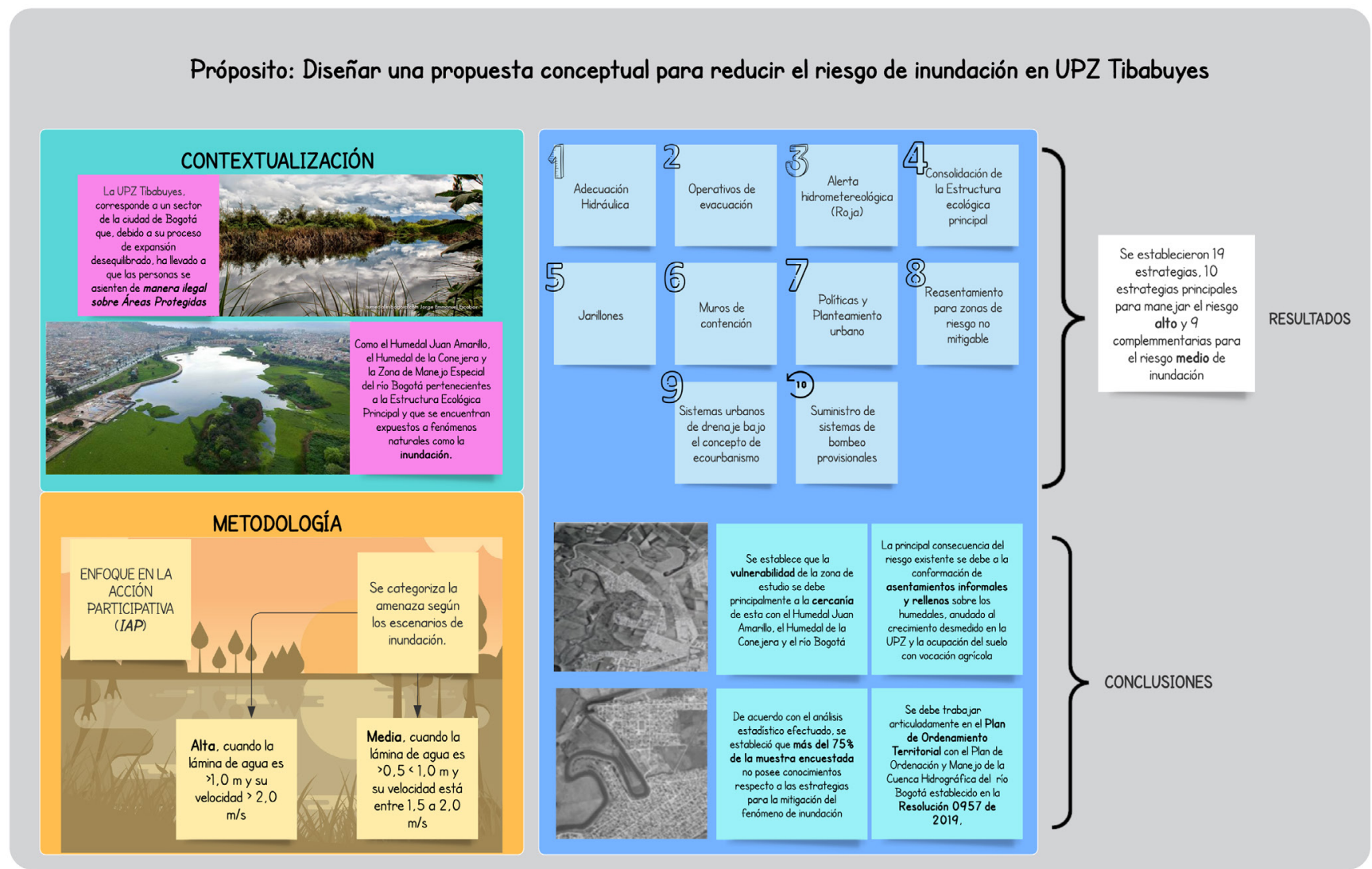

Figura 1. Resumen gráfico. Fuente: Autores.

\section{INTRODUCCIÓN}

El crecimiento urbano de las ciudades de Colombia ha sido consecuencia de distintos procesos de expansión, como aquellos relacionados con el crecimiento poblacional; las migraciones interdepartamentales en la búsqueda de nuevas oportunidades económicas y sociales; entre otros. Durante la década de los años cincuenta, el crecimiento demográfico en Bogotá había incrementado en un $250 \%$ con respecto a los datos poblacionales del año 1819 (Gobernación de Cundinamarca, 2017), donde, al ser la capital del país, tenía un escenario propicio para el desarrollo económico e industrial, generando un incremento en la ocupación del suelo y afectando las zonas rurales, cuerpos de agua y ecosistemas como consecuencia de la alta demanda de urbanización y de infraestructura vial; además de la aparición de asentamientos informales en la periferia, que forjaron patrones de expansión no planificados que llevaron a un desarrollo ecológicamente insostenible y que generó un cambio en las dinámicas urbanas (Guzmán, 2015).

Debido a la necesidad de la organización territorial del Distrito Capital, surgieron lineamientos en su división administrativa, tales como el Acuerdo 11 de 1954 por alcaldías menores de municipios anexos, posteriormente se dividió en diecinueve alcaldías con el Acuerdo 8 de 1977. Para el año 1993, el Decreto de Ley 421 en su artículo 62, establece las localidades como entes territoriales dentro de la división político-administrativa del Distrito Capital. Por último, el Artículo 49 del Decreto 190 de 2004 establece en el Plan de Ordenamiento Territorial, dividir la ciudad en la "Unidades de Planeamiento Zonal" (UPZ) para asignar las normas urbanísticas de las veinte localidades de la ciudad. 
La localidad de Suba limita al norte con el municipio de Chía, al occidente con el de Cota, (teniendo al río Bogotá de por medio en ambos límites), al oriente con la localidad de Usaquén, y al sur con las localidades de Engativá y Barrios Unidos, (SDP, 2017). Esta localidad se conforma de 11 UPZ. En el caso de la UPZ Tibabuyes, se localiza en el extremo suroccidental, con una extensión de 726,4 ha, equivalentes al $11,6 \%$ del área total de las UPZ de la localidad. (SDP, 2017); presenta asentamientos de manera ilegal sobre las áreas protegidas, donde se ubican el Humedal Juan Amarillo, el Humedal de la Conejera y la Zona de Manejo Especial del Río Bogotá, pertenecientes a la Estructura Ecológica Principal (SDP, 2017). Debido a la presencia de estos sistemas hídricos, la zona se encuentra expuesta a fenómenos naturales como la inundación.

La Alcaldía Mayor de Bogotá indica que, en el año 2005, el crecimiento de Bogotá se concentró en los extremos Occidental, Sur y Norte de la ciudad, específicamente hacia las localidades de San Cristóbal, Bosa, Kennedy, Suba y Engativá, debido al surgimiento de pequeños asentamientos legalizados y conexos a desarrollos urbanísticos como el Rincón de Santa Inés y Tibabuyes, en Suba.

Conjuntamente, la zona suroccidental de la localidad de Suba ha dejado de ser una zona agrícola para convertirse en un sector suburbanizado, que posee características de suelos rurales como la vegetación, la capacidad de filtración y almacenamiento del agua que, por consecuencia del cambio en el uso del suelo actual, genera un aumento en los niveles de escorrentía (Serrano y Hernández, 2012).

\section{Ronda de inundación y estrategias del plan de acción.}

La UPZ Tibabuyes la constituyen los barrios Tibabuyes II, Sabana de Tibabuyes y Rincón de Santa Inés, entre otros, y presentan mayor riesgo de inundación, debido a que se encuentran por debajo del nivel promedio de los cuerpos de agua, del Humedal de la Conejera y, en el caso de Lisboa, Santa Cecilia, Santa Rita de Suba y San Pedro, se encuentran por debajo del Humedal Juan Amarillo y próximos a la ronda del Río Bogotá (Serrano \& Romero, 2017). La urbanización desmedida sobre estos cuerpos de agua ha generado alteraciones del cauce del río Bogotá y del Humedal Juan Amarillo, aumentando la probabilidad de ocurrencia de encharcamientos entre el 10 y el 65\%. (IDIGER, 2018).

Según la Resolución 1631 de 2018, estos barrios poseen zonas en riesgo medio y alto de inundación debido a que se encuentran en el área mínima potencialmente inundable de la cuenca media del río Bogotá, que equivale a 8920 Hectáreas (IDIGER, 2018). Adicionalmente, el Instituto de Hidrología, Meteorología y Estudios Ambientales (IDEAM) pronostica que, para el periodo 2011-2040, la UPZ Tibabuyes presentará un exceso de precipitaciones mayor a un $40 \%$ (IDIGER, s. f.) lo cual proyecta otro factor de riesgo para los barrios susceptibles ante la ocurrencia de inundaciones.

Una de las acciones que pretenden mitigar las situaciones agravantes del riesgo de inundación es la limpieza del cauce del río Bogotá, por lo que se han realizado trabajos de limpieza por parte de la Alcaldía Local de Suba y la comunidad. Estas jornadas, han demostrado fallas en los jarillones, presencia de escombros, residuos sólidos y obstrucciones. De la misma manera, la Corporación Autónoma Regional de Cundinamarca (CAR) para el año 2013 invirtió en obras públicas, en la limpieza de los jarillones, además de establecer muros de contención y estaciones de monitoreo (Alcaldía Local de Suba, 2012).

Desde el año 2017, la Unidad Nacional para la Gestión del Riesgo de Desastres (UNGRD) con 
el apoyo del IDEAM, en el marco del Plan Nacional de Gestión del Riesgo, han elaborado lineamientos para la evaluación probabilística del riesgo por inundación en todo el país. (UNGRD, 2018); sin embargo, a pesar de las acciones realizadas por las instituciones para la mitigación del riesgo por inundación, aún falta fortalecer las estrategias preventivas $y$ correctivas en todo el territorio colombiano, y determinar los escenarios más susceptibles de riesgo, haciendo un análisis articulado en las diferentes escalas zonales, como las Unidades de Planeamiento Zonal, donde se pueden evidenciar más fácilmente varios factores de riesgo y las afectaciones a un mayor nivel. Para el caso de estudio de la UPZ Tibabuyes, aparte de los escenarios mencionados anteriormente, también se considera que varios barrios pertenecientes a esta UPZ presentan problemas en el sistema de alcantarillado, los cuales se ven desfavorecidos por la descarga libre y el aumento en los niveles del río Bogotá.
Con este panorama, se establece como objetivo general del trabajo de investigación: diseñar una propuesta conceptual para la reducción del impacto de las inundaciones en la UPZ Tibabuyes.

\section{MATERIALES Y MÉTODOS}

El tipo de investigación es descriptiva con un enfoque de acción participativa el cual establece medidas de planificación para dar solución a una problemática a partir de la intervención social de los actores involucrados.

La metodología empleada se dividió en tres fases (Figura 2). La primera fase corresponde al diagnóstico del área de estudio; en la segunda fase se realizó la evaluación de las estrategias de mitigación a través de la percepción; y en la última fase, se desarrolló el plan de acción como solución al riesgo.

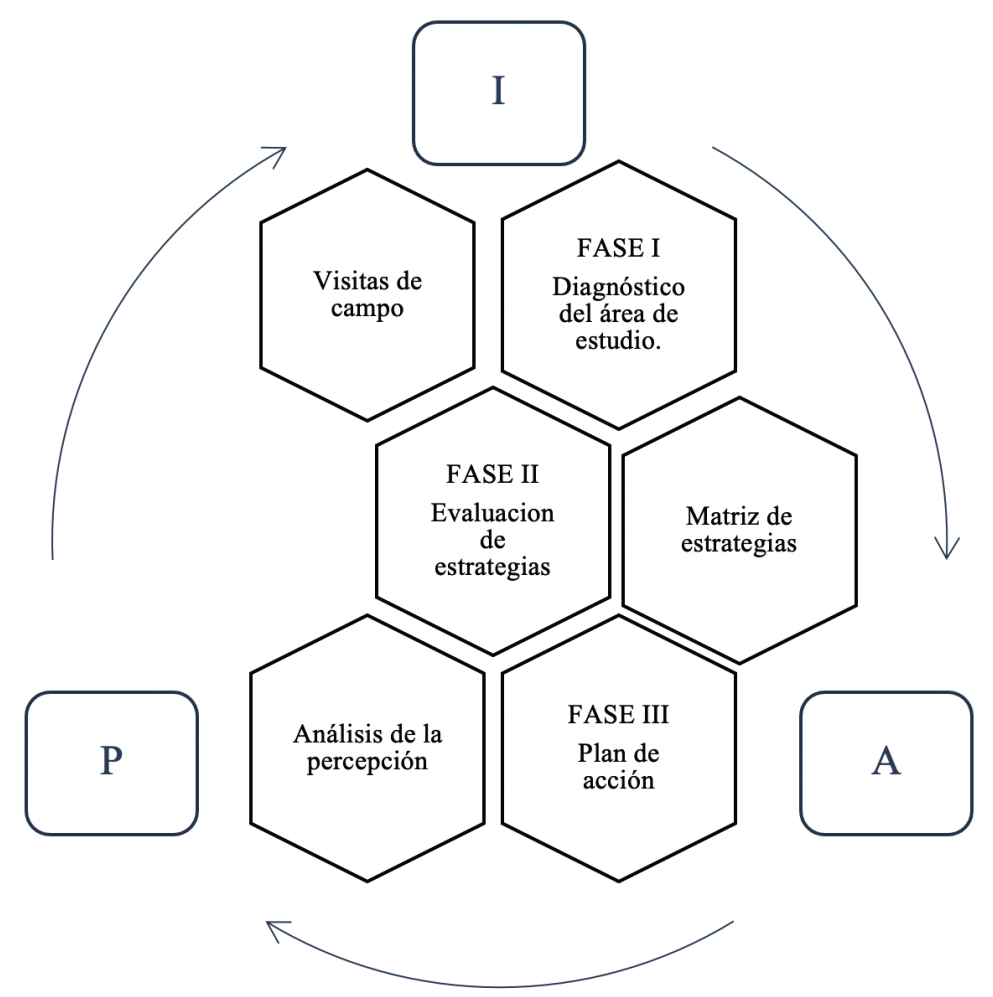

Figura 2. Fases para el desarrollo de la investigación.

Fuente: Autores. 
En la primera fase, se realizó el diagnóstico del área de estudio con el fin de hacer un reconocimiento de la zona; en esta fase se incorpora un marco legal, por otra parte, se realizó un estudio de ocupación histórica del suelo a través del uso de aerofotografías obtenidas del Instituto Geográfico Agustín Codazzi (IGAC), se evaluaron las dinámicas poblacionales, las condiciones económicas de la zona y se identificaron las problemáticas usando como instrumento principal fuentes de información secundaria. En la segunda fase, se planteó una matriz (fig. 2) de evaluación la cual cuenta con las medidas establecidas por el Instituto Distrital de Gestión de Riesgo y Cambio Climático (IDIGER) para el periodo de lluvias Año 2019 para la mitigación del riesgo por inundación y un indicador basado en el concepto de Amenaza Alta y Amenaza media de inundación la cual toma variables de profundidad y velocidad de flujo de cauce. Cada medida fue clasificada según el tipo de Amenaza por mitigar; esta clasificación se realizó con base en información secundaria.

Así mismo, en esta fase se realizó el análisis de percepción de la vulnerabilidad a la población como forma de valoración para la matriz planteada. Para estimar la percepción de las personas, se llevó a cabo un sistema de recolección de información a partir de una encuesta con 27 preguntas con respuesta cerrada relacionadas con las medidas presentadas en la matriz de evaluación; la encuesta fue aplicada a una muestra significativa, que se definió a través de un muestreo estadístico estratificado, dividiendo la población de la Unidad de Planeamiento Zonal Tibabuyes por estratos (Sin estrato, estrato 2 y estrato 3) y a partir de esto se determinó la muestra total. Para la selección de la muestra se utilizó la siguiente fórmula estadística:
Ecuación 1. Fórmula para establecer el tamaño de la muestra (n).

En donde:

N: Tamaño de la población.

k: Constante que depende del nivel de confianza (90\%).

e: Error muestral deseado.

p: Es la proporción de individuos que poseen en la población la característica de estudio. q: Es la proporción de individuos que no poseen esa característica, es decir, 1 - P. $\mathrm{n}$ : Es el tamaño de la muestra.

Con base en la muestra significativa de 277 personas, los resultados se relacionaron directamente con las estrategias planteadas para la mitigación del riesgo; después, se realizó un análisis estadístico descriptivo utilizando como base el Software Estadístico R, en el cual se determinó la aceptación del valor central de la distribución de acuerdo con el grado de aceptación y así tomar las medidas de centralización por medio de medidas de posición.

En la tercera fase metodológica, se elaboró un plan de acción conformado por 19 estrategias de acción, que se enfocan directamente con cada medida planteada en la matriz (fig.2) de evaluación. Dentro del plan de acción se establecen los objetivos, los impactos generados, las actividades para realizar la estrategia, las recomendaciones técnicas y el valor presupuestado con las fuentes de financiación con el fin de proponer una solución al riesgo existente en la UPZ Tibabuyes.

$$
n=\frac{k^{2} * \mathrm{p} * \mathrm{k} * \mathrm{~N}}{\left(e^{2} *(N-1)+K^{2} * P * Q\right.}
$$




\section{RESULTADOS Y DISCUSIÓN Diagnóstico del área de estudio}

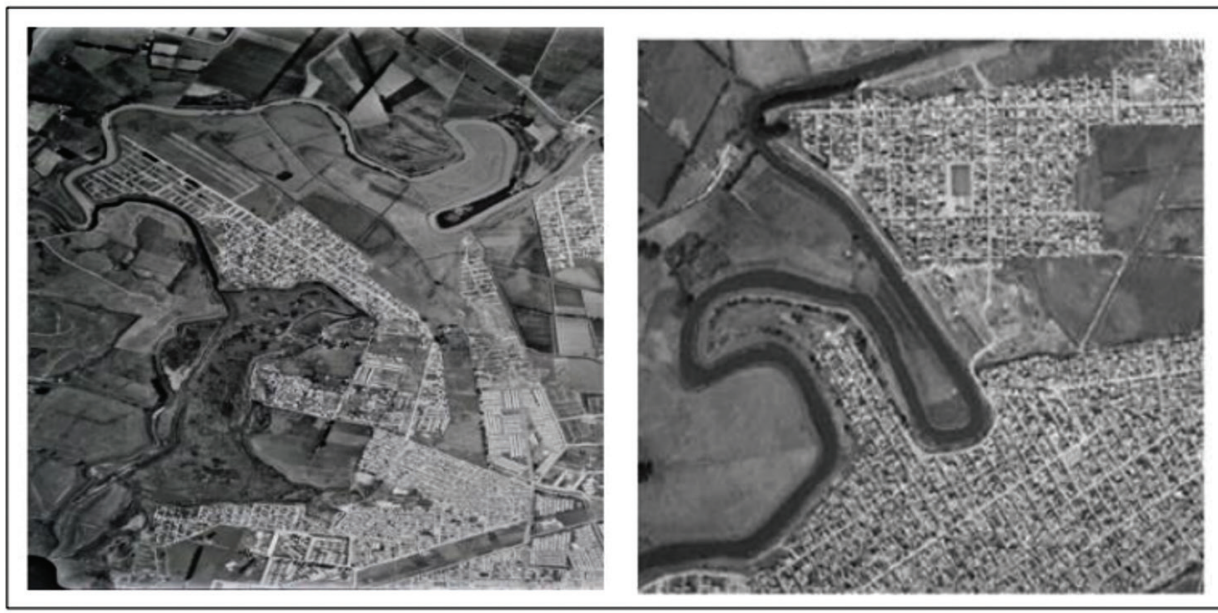

Figura 3. Aerofotografías de la UPZ Tibabuyes para el año 1995 y 2004.

Fuente: Instituto Geográfico Agustín Codazzi (IGAC,2019)

La zona de estudio se ha ido conforman- desarrollo socioeconómico y ambiental de la do bajo un marco legal que ha permitido el UPZ (Tabla 1).

Tabla 1. Matriz de cumplimiento de la normativa asociada con la UPZ Tibabuyes.

\begin{tabular}{l|c|c}
\hline Norma & Si cumple & No cumple \\
\hline Decreto 190 de 2004 & $X$ & $X$ \\
Ley 1523 de 2012 & $X$ & \\
Ley 99 de 1993 & & \\
Decreto Distrital 657 de 1994 & & $X$ \\
Decreto 566 de 2014 & & $X$ \\
Ley 810 de 2003 & $X$ & $X$ \\
Acuerdo 17 de 2009 & $X$ & $X$ \\
Acuerdo Local 4 de 2016 & \\
Decreto 62 de 2006 & \\
Resolución 957 de 2019 & & \\
\hline
\end{tabular}

Fuente: Autores

Es de resaltar que la localidad de Suba ha desarrollado múltiples iniciativas ciudadanas alrededor de los humedales de La Conejera, Córdoba, Juan Amarillo y Torca. De igual for$\mathrm{ma}$, se han presentado intervenciones y actividades en el río Bogotá, la Quebrada Salitrosa, los Cerros de Suba y las UPZ con mayor grado de urbanización (Rodríguez \& Guarín, 2015).
También se han presentado iniciativas en la Comisión Ambiental Local de Suba en donde se han adelantado procesos de protección de zonas periféricas y rondas de los humedales y del río Bogotá, se ha hecho la recuperación del espacio público, campañas de sensibilización y acciones encaminadas a la recuperación de los cerros de Suba (Rodríguez \& Guarín, 2015). 
En cuanto a las dinámicas poblacionales para la Localidad de Suba en los años 2014-2017, se estimó que la UPZ Tibabuyes sería la segunda más poblada con un porcentaje equivalente al $20.7 \%$ (SDP, 2017).

Tabla 2. Proyecciones de población en la Localidad de Suba 2016-2020.

\begin{tabular}{|c|c|}
\hline Años & Total \\
\hline 2016 & 1.250 .734 \\
2017 & 1.282 .978 \\
2018 & 1.315 .509 \\
2019 & 1.348 .372 \\
2020 & 1.381 .597 \\
\hline
\end{tabular}

Fuente: Adaptación Secretaría Distrital de Planeación (2014)

Con respecto a las proyecciones estimadas para la Localidad de Suba entre los años 2016- 2020, la población aumentará (Tabla 2), lo que también podría explicar el aumento de asentamientos $y$, por ende, el impacto sobre la ronda hídrica del río Bogotá, de conformidad con la ubicación de algunas viviendas en las UPZ El Rincón y Tibabuyes, con estratificación 1 y 2 respectivamente (SDP, 2017). Otra situación que se podría dar es el crecimiento de actividades económicas informales, que no cumplirían normas ambientales relacionadas con vertimientos o residuos sólidos cerca a los cuerpos hídricos de interés.

Según la Alcaldía Mayor de Bogotá, la UPZ cuenta con un buen Índice de Calidad de Vida (ICV), este se basa en la aproximación al cálculo del índice, a partir del principio de que todas las dimensiones o núcleos tienen el mismo peso $y$, dentro de cada dimensión, todos los indicadores también tienen el mismo peso. Esta aproximación se ha usado ampliamente en la construcción de índices multidimensionales como la medición multidimensional de la pobreza (Alkire y Foster, 2011; Alkire y Santos 2010; Angulo, Díaz y Pardo, 2011). En este sentido, para obtener el puntaje de cada dimensión, se promedian todas las variables (ya normalizadas a la escalada de 0 a 100) que contienen esa dimensión; después, para calcular el ICV Urbana se promedia el puntaje de las cuatro dimensiones o núcleos, esta están determinadas en Necesidades básicas, dotación Urbana y medio ambiente, bienestar y oportunidades y cohesión social., al tener 93.1 puntos de 100 posibles; sin embargo, cuenta con el $8.1 \%$ de la población que vive en situación de pobreza. Así mismo, con base en el POT de Bogotá, el uso del suelo urbano de Suba se divide en siete campos de actividad, de los cuales se destaca el uso residencial (el más predominante); permitiéndose, en bajas cantidades, el comercio y los servicios (Instituto de Estudios Urbanos, 2011). En la UPZ Tibabuyes solo se presentan tres de estas (sin estrato, 2 y 3) (Secretaria Distrital de Planeación, 2017).

Con relación a la ocupación de sus habitantes, la mayoría se dedica a trabajar en construcción, mecánica, conducción, cultivos de flores, servicio doméstico, restaurantes, operación de fábricas y el sector informal; esto se ve más presente en zonas como Casa Blanca, Rincón, Tibabuyes y Suba Centro (Instituto de Estudios Urbanos, 2011). 
Evaluación de las estrategias para los escenarios de riesgo

Las estrategias para la mitigación del riesgo por inundación fueron adaptadas a partir de las acciones establecidas en el Plan de Contingencia elaborado por el Instituto Distrital de Riesgos y Cambio Climático (IDIGER).

Según los escenarios planteados, se construyó un indicador basado en el concepto de Riesgo alto y Riesgo medio, que toma las variables de profundidad y velocidad del flujo del cauce. Para la Amenaza Alta de inundación, la profundidad de lámina de agua debe oscilar entre $1,5 \mathrm{~m} / \mathrm{s}$ y $1,0 \mathrm{~m} / \mathrm{s}$ (Figura 2), según la metodología de inundación hidráulica de "Bogotá MEJOR para Todos", 2016-2020, propuesta por el IDIGER.

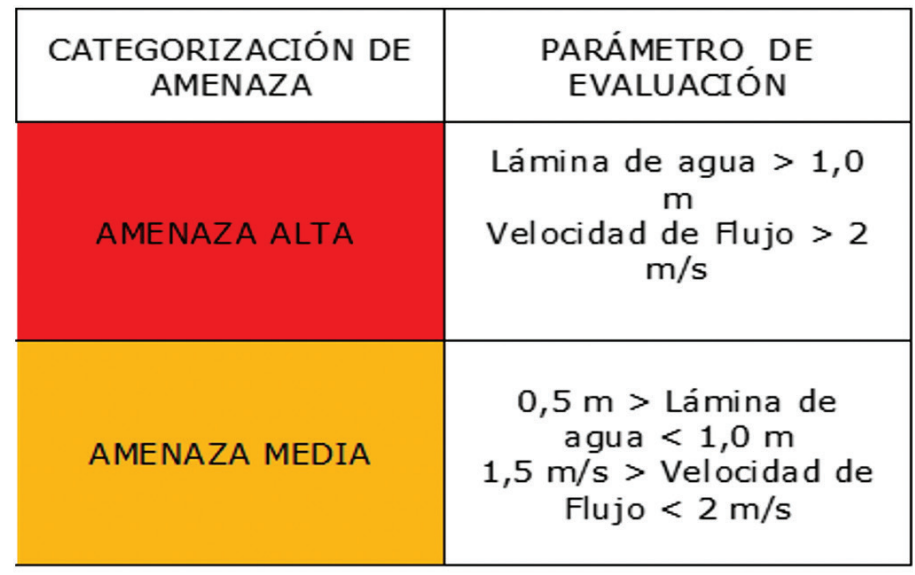

Figura 3. Categorización de la amenaza de acuerdo con los escenarios de inundación.

Fuente: Autores.

Se elaboró la matriz de estrategias, que presenta 19 medidas establecidas en caso de presentarse riesgo alto y medio de inundación. Para el riesgo alto de inundación, se establecieron medidas de tipo estructural como jarillones, muros de contención, adecuación hidráulica e implementación de Sistemas Urbanos de Drenaje (SUDS), además de otro tipo de medidas no estructurales como operativos de evacuación, alertas hidrometereológicas, reasentamiento, políticas sobre la planeación urbana, consolidación de la Estructura Ecológica Principal (EEP) y sistemas de bombeo provisionales.

Para el riesgo medio de inundación, se encuentran estrategias de tipo no estructural como Sistemas de Alerta Temprana por eventos hidrológicos extremos; capacitaciones y sensibilizaciones sobre el riesgo; mantenimiento de los cuerpos de agua y del mobiliario urbano; modelación hidrológica; monitoreo de Iluvias; monitoreo del caudal del rio; y señalización preventiva.

La matriz fue diseñada con el fin de evaluar, a través de la percepción de los habitantes de la zona, la seguridad, el conocimiento y la eficacia de este tipo de medidas en caso de presentarse algún tipo de escenario anteriormente mencionado.

\section{Percepción del riesgo}

El análisis de la percepción fue realizado a partir de los resultados estadísticos que se presentan en cuatro apartados:

APARTADO I: evaluación general de la percepción de los habitantes de la zona de estudio. 


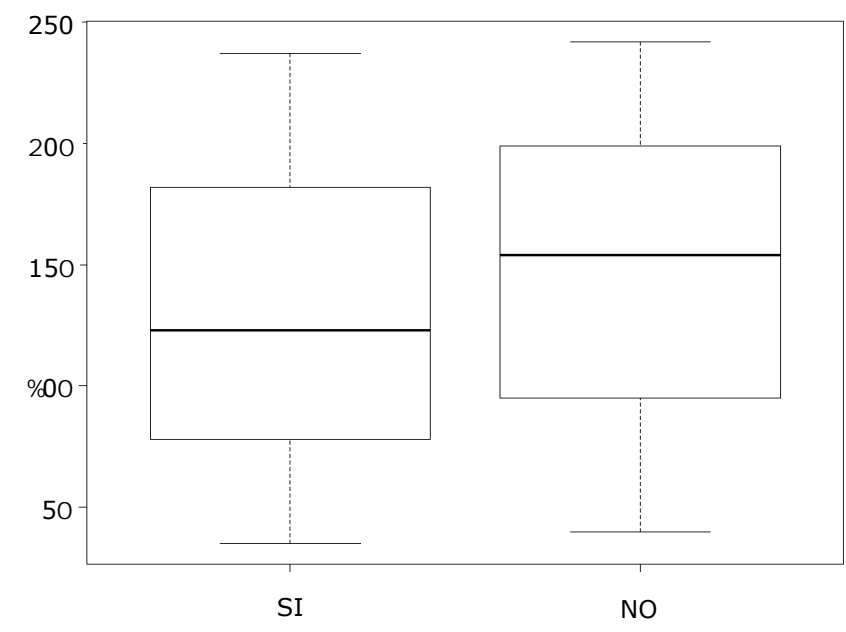

Figura 4. Gráfico Bloxplot sobre la percepción de las estrategias de mitigación del riesgo.

Fuente: Autores

Según la Figura 4, se presenta un gráfico Boxplot en el cual se comparan dos categorías de respuesta lógicas (sí y no); la distribución de ambas respuestas fue evaluada a partir de una encuesta para identificar la percepción de la población en relación con el conocimiento, el sentimiento de seguridad y la efectividad de las estrategias para la mitigación del riesgo de inundación y su importancia.

Los resultados de la estadística descriptiva general demuestran que los rangos intercuartílicos, con los valores de ambas respuestas, representan el 50\% comprendido en 104 encuestados, que siendo esta, la diferencia entre los valores del tercer y primer cuartil; sin embargo, los valores de la mediana son mayores para las respuestas "No" que las respuestas "Sí", con los resultados de 154 y 123, respectivamente. Lo que confirma el resultado establecido en el Boxplot.

Para este análisis, el indicador más importante (aunque cada vez más cuestionado) es el llamado $\mathrm{p}$-valor, mediante el que, habitualmente, se considera estadísticamente significativo el efecto del tratamiento cuando este es inferior a 0,05. En este caso, el p-valor es de 2,2e-16, por lo que se consideraría que existe diferencia significativa entre los grupos; por ende, se estima que la población no se siente segura con las medidas implementadas actuales, y requieren la implementación de un plan de gestión de riesgo.De acuerdo con Vallejo y Vélez (2009), el conocimiento del riesgo se adquiere en mayor parte mediante los medios informativos y no de la experiencia directa, es por esto que la percepción del riesgo y los rasgos de vulnerabilidad varían teniendo en cuenta el medio informativo y la difusión de los mismos.

APARTADO II: análisis de la percepción basado en el conocimiento de las estrategias para la mitigación del riesgo.

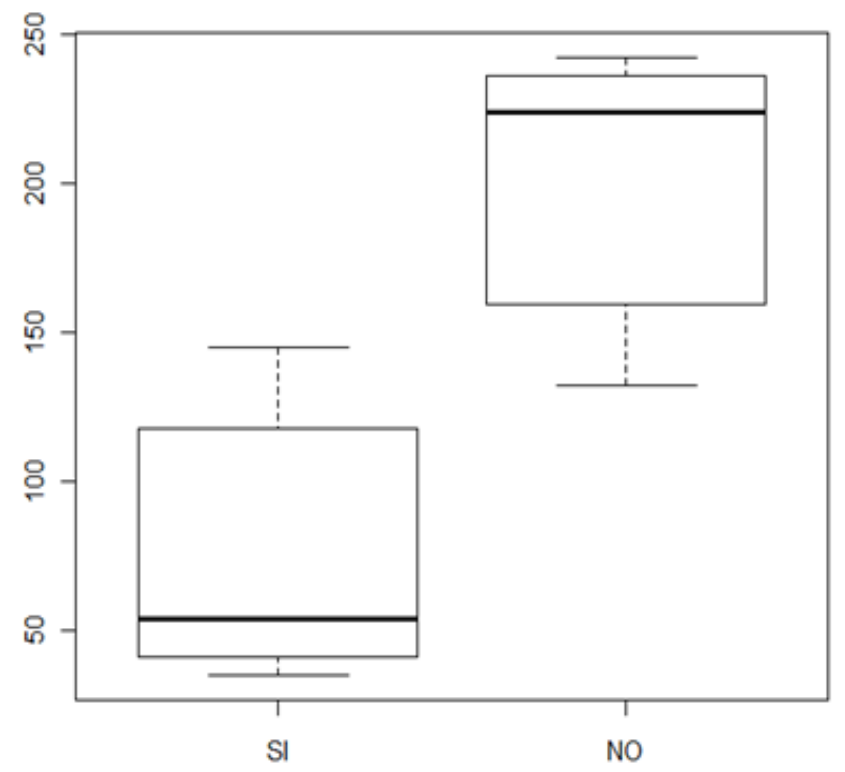

Figura 5. Conocimiento de los habitantes sobre las restricciones y determinación de riesgo de inundación.

Fuente: Autores.

A través del Bloxplot presentado en la Figura 5, se visualiza la gran dispersión presentada entre las respuestas, lo que sugiere que existe desconocimiento de este tipo de estrategias, puesto que más del $75 \%$ de los habitantes confirman tener desconocimiento, según los resultados del tercer cuartil de la respuesta "No". 
De igual forma, se confirma que los rangos intercuartílicos están comprendidos en 68,6 , que es la diferencia entre los valores del tercer y primer cuartil; sin embargo, los valores de la mediana son mayores para las respuestas "No" que las respuestas "Sí", con los resultados de 223,5 y 53,5 , respectivamente, lo que confirma los resultados del Boxplot.

Respecto a la percepción de los habitantes de la UPZ Tibabuyes encuestados, existe desconocimiento en cuanto al Plan de Emergencia. Así mismo, las personas desconocen las señales de prevención del riesgo; sin embargo, de acuerdo con la visita de campo realizada, se encontró que en el barrio Villa Cindy existe la presencia de un cartel informativo sobre asentarse en lugares en zona de riesgo, menos de la mitad de la muestra afirma conocer el mapa de riesgo de la zona. De igual manera, existe desconocimiento sobre las alertas tempranas, que, de acuerdo con la muestra encuestada, no han sido implementadas.

Contrariamente, las personas conocen las restricciones de encontrarse en una zona de riesgo, sin embargo, debido a factores económicos y al arraigo sobre sus viviendas, hace que no pretendan trasladarse a otro lugar. Así mismo, algunas personas encuestadas afirman haber asistido a capacitaciones sobre el riesgo; sin embargo, en la mayoría de los barrios no existió ninguna información acerca de esto. Finalmente, la población ha presenciado campañas de limpieza del río y humedales, a nivel general, afirmando su participación en estos eventos seis años atrás, lo que sugiere que la limpieza de este tipo de cuerpos de agua no es relevante, y se pueden presentar otro tipo de complicaciones relacionadas con la cantidad de sedimentos que obstruyen el drenaje.

\section{Plan de acción como forma de mitigación del riesgo}

Se proponen una serie de estrategias de acción en escenarios de riesgo alto y medio de inundación de acuerdo con cada estrategia evaluada, con el fin de garantizar su eficacia a la hora de presentarse un riesgo de inundación.

El plan de acción consta de 19 estrategias, de las cuales 10 corresponden a estrategias implementadas en caso de presentarse un escenario de riesgo alto de inundación, y las 9 estrategias complementarias deben ser empleadas en caso de presentarse un escenario de riesgo medio de inundación (Tabla 3- Tabla 4), la descripción específica de cada estrategia puede visualizarse en el Anexo "PROPUESTA CONCEPTUAL PARA LA MITIGACIÓN DE RIESGO POR INUNDACIÓN EN LA UNIDAD DE PLANEAMIENTO ZONAL(UPZ) TIBABUYES, LOCALIDAD DE SUBA".

Así, las 10 estrategias implementadas en escenarios de riesgo alto se resumen de la siguiente forma: adecuación hidráulica en el tramo del $\mathrm{Km} 68$ con la ampliación de 30 a 60 metros del cauce, operativos de evacuación de acuerdo con los planes de emergencia, alerta hidrometereológica roja por riesgo crítico de desbordamiento en el río, consolidación de la estructura ecológica principal, levantamiento y mantenimiento de Jarillones sobre el borde izquierdo del afluente, implementación de muros de contención, desarrollo de normativas que regulen el uso del suelo, reasentamiento para zonas de riesgo no mitigable, implementación de sistemas urbanos de drenaje bajo el concepto de ecourbanismo y suministro de sistemas de bombeo provisionales.

Mientras que las estrategias complementarias son: instalar sistemas de alertas por eventos hidrológicos externos, declarar el sistema de alerta naranja cuando se identifique la tendencia ascendente de los niveles de los ríos y la persistencia de las lluvias, programas de capacitación sobre el riesgo y talleres sobre el manejo de la emergencia, retiro de residuos y estructuras que afecten el drenaje, realizar 
mantenimiento con el fin de eliminar obstrucciones que puedan generar fallas en el drenaje, modelación hidrológica, monitoreo de
Iluvias, monitoreo del afluente y del caudal del río, redes comunitarias que alerten el riesgo con el fin de resguardarse.

Tabla 3. Matriz de estrategias riesgo alto.

\begin{tabular}{|c|c|c|}
\hline \multicolumn{3}{|c|}{$\begin{array}{l}\text { MATRIZ DE ESTRATEGIAS PARA LA MITIGACIÓN DE RIESGO POR INUNDACIÓN } \\
\text { (Intervención correctiva-prospectiva ) }\end{array}$} \\
\hline Tipo de estrategia & Estrategia & Indicador \\
\hline Adecuación hidráulica & $\begin{array}{c}\text { La adecuación hidráulica consiste en la intervención de un } \\
\text { tramo de } 68 \mathrm{Km} \text { desde Puente la Virgen hasta las compuertas } \\
\text { de Alicachín, en el cual se genera el mejoramiento del afluente y } \\
\text { la ampliación de } 30 \text { a } 60 \mathrm{mts} \text { del cauce. }\end{array}$ & $\begin{array}{l}\text { Profundidad }(\mathrm{H})>1 \mathrm{~m} \\
\text { Velocidad de Flujo }(\mathrm{Vf})>2 \mathrm{~m} / \mathrm{s}\end{array}$ \\
\hline Operativos de evacuación & $\begin{array}{c}\text { Se establecen operativos según los planes de emergancia en los } \\
\text { cuales se tiene en cuenta lugares de resguardo y atención a las } \\
\text { necesidades básicas de las personas }\end{array}$ & $\begin{array}{l}\text { Profundidad }(\mathrm{H})>1 \mathrm{~m} \\
\text { Velocidad de Flujo }(\mathrm{Vf})>2 \mathrm{~m} / \mathrm{s}\end{array}$ \\
\hline $\begin{array}{l}\text { Alerta hidrometereológica } \\
\qquad \text { (ROJA) }\end{array}$ & $\begin{array}{c}\text { Sistema de alerta roja por riesgo crítico de desbordamiento por } \\
\text { el aumento del nivel del río o cuando ha empezado la } \\
\text { inundación. }\end{array}$ & $\begin{array}{l}\text { Profundidad }(\mathrm{H})>1 \mathrm{~m} \\
\text { Velocidad de Flujo }(\mathrm{Vf})>2 \mathrm{~m} / \mathrm{s}\end{array}$ \\
\hline $\begin{array}{l}\text { Consolidación de la Estructura } \\
\text { Ecológica Principal }\end{array}$ & $\begin{array}{l}\text { Generación de un corredor biológico de ronda con el fin de } \\
\text { generar la rehabilitación de humedales que permitan la } \\
\text { regulación hídrica y otros servicios ambientales. }\end{array}$ & $\begin{array}{l}\text { Profundidad }(\mathrm{H})>1 \mathrm{~m} \\
\text { Velocidad de Flujo }(\mathrm{Vf})>2 \mathrm{~m} / \mathrm{s}\end{array}$ \\
\hline Jarilllones & $\begin{array}{l}\text { Levantamiento y mantenimiento de jarillones ubicados sobre el } \\
\text { borde izquierdo del afluente. }\end{array}$ & $\begin{array}{l}\text { Profundidad }(\mathrm{H})>1 \mathrm{~m} \\
\text { Velocidad de Flujo }(\mathrm{Vf})>2 \mathrm{~m} / \mathrm{s}\end{array}$ \\
\hline Muros de contención & $\begin{array}{l}\text { Implementación de muros de contención elaborados con } \\
\text { sedimentos de los cuerpos de agu con duración de } 100 \text { años. }\end{array}$ & $\begin{array}{l}\text { Profundidad }(\mathrm{H})>1 \mathrm{~m} \\
\text { Velocidad de Flujo }(\mathrm{Vf})>2 \mathrm{~m} / \mathrm{s}\end{array}$ \\
\hline Politicas y Planeamiento Urbano & $\begin{array}{c}\text { Desarrollo de normativas que regulen el uso del suelo y el tipo } \\
\text { de edificación y en consecuencia la realización de } \\
\text { planeamientos urbanos que tengan en cuenta las zonas con } \\
\text { riesgo de inundación. }\end{array}$ & $\begin{array}{l}\text { Profundidad }(\mathrm{H})>1 \mathrm{~m} \\
\text { Velocidad de Flujo }(\mathrm{Vf})>2 \mathrm{~m} / \mathrm{s}\end{array}$ \\
\hline $\begin{array}{l}\text { Reasentamiento para zonas de riesgo } \\
\text { no mitigable }\end{array}$ & $\begin{array}{c}\text { Se realiza un monitoreo de predios ocupados de forma ilegal, } \\
\text { aquellos que se encuentran en riesgo deben ser reubicados. El } \\
\text { IDIGER compra este tipo de predios y se genera la enajenación } \\
\text { voluntaria. }\end{array}$ & $\begin{array}{c}\text { Profundidad }(\mathrm{H})>1 \mathrm{~m} \\
\text { Velocidad de Flujo }(\mathrm{Vf})>2 \mathrm{~m} / \mathrm{s}\end{array}$ \\
\hline $\begin{array}{l}\text { Sistemas urbanos de drenaje bajo el } \\
\text { concepto de ecourbanismo }\end{array}$ & $\begin{array}{c}\text { Implementación de parques lineales sobre el borde del río, } \\
\text { adoquines permeables y sistemas que permitan la captación de } \\
\text { agua. }\end{array}$ & $\begin{array}{c}\text { Profundidad }(\mathrm{H})>1 \mathrm{~m} \\
\text { Velocidad de Flujo }(\mathrm{Vf})>2 \mathrm{~m} / \mathrm{s}\end{array}$ \\
\hline $\begin{array}{l}\text { Suministro de sistemas de bombeo } \\
\text { provisionales }\end{array}$ & $\begin{array}{c}\text { El IDIGER suministra motobombas y electrobombas los cuales } \\
\text { permiten el manejo de inundaciones. }\end{array}$ & $\begin{array}{c}\text { Profundidad }(\mathrm{H})>1 \mathrm{~m} \\
\text { Velocidad de Flujo }(\mathrm{Vf})>2 \mathrm{~m} / \mathrm{s}\end{array}$ \\
\hline
\end{tabular}


Mientras que las estrategias complementarias son: instalar sistemas de alertas por eventos hidrológicos externos, declarar el sistema de alerta naranja cuando se identifique la tendencia ascendente de los niveles de los ríos y la persistencia de las lluvias, programas de capacitación sobre el riesgo y talleres sobre el manejo de la emergencia, retiro de residuos y estructuras que afecten el drenaje, realizar mantenimiento con el fin de eliminar obstrucciones que puedan generar fallas en el drenaje, modelación hidrológica, monitoreo de lluvias, monitoreo del afluente y del caudal del río, redes comunitarias que alerten el riesgo con el fin de resguardarse.

\section{Dentro de las estrategias se especifican los objetivos, la población beneficiada, los reque- rimientos técnicos, el presupuesto, las activi- dades a realizar y las fuentes de financiación.}

Tabla 4. Matriz de estrategias Riesgo medio.

\begin{tabular}{|c|c|c|}
\hline \multicolumn{3}{|c|}{$\begin{array}{l}\text { MATRIZ DE ESTRATEGIAS PARA LA MITIGACIÓN DE RIESGO POR INUNDACIÓN } \\
\text { (Intervención correctiva-prospectiva ) }\end{array}$} \\
\hline Tipo de estrategia & Estrategia & Indicador \\
\hline $\begin{array}{c}\text { Sistemas de alertas tempranas por } \\
\text { eventos hidrológicos } \\
\text { extremos }\end{array}$ & $\begin{array}{c}\text { Instalar sistemas de alertas por eventos } \\
\text { hidrológicos extremos en zonas que han } \\
\text { presentado eventos históricos recurrentes con el fin } \\
\text { de evitar desbordamiento de los cauces. }\end{array}$ & $\begin{array}{l}0,5 \mathrm{~m}>\text { Profundidad }(\mathrm{H})<1,0 \mathrm{~m} \\
\text { Velocidad de Flujo }(\mathrm{Vf})<2 \mathrm{~m} / \mathrm{s}\end{array}$ \\
\hline $\begin{array}{l}\text { Alerta hidrometereológica } \\
\text { (NARANJA) }\end{array}$ & $\begin{array}{c}\text { Sistema de alerta naranja la cual se declara cuando } \\
\text { la tendencia ascendente de los niveles de los ríos y } \\
\text { la persistencia de las lluvias indican la posibilidad } \\
\text { de que se presenten desbordamientos en las } \\
\text { próximas horas. }\end{array}$ & $\begin{array}{l}\text { Profundidad }(\mathrm{H})>1 \mathrm{~m} \\
\text { Velocidad de Flujo }(\mathrm{Vf})>2 \mathrm{~m} / \mathrm{s}\end{array}$ \\
\hline $\begin{array}{l}\text { Capacitación y sensibilización a la } \\
\text { comunidad sobre el riesgo }\end{array}$ & $\begin{array}{c}\text { Programas de capacitación sobre el riesgo y talleres } \\
\text { sobre el manejo de la emergencia. }\end{array}$ & $\begin{array}{l}0,5 \mathrm{~m}>\text { Profundidad }(\mathrm{H})<1,0 \mathrm{~m} \\
\text { Velocidad de Flujo }(\mathrm{Vf})<2 \mathrm{~m} / \mathrm{s}\end{array}$ \\
\hline $\begin{array}{l}\text { Limpieza de los cuerpos de agua } \\
\text { presentes en la zona }\end{array}$ & $\begin{array}{l}\text { Retiro de residuos y estructuras que afecten el } \\
\text { drenaje del cuerpo de agua. }\end{array}$ & $\begin{array}{l}0,5 \mathrm{~m}>\text { Profundidad }(\mathrm{H})<1,0 \mathrm{~m} \\
\text { Velocidad de Flujo }(\mathrm{Vf})<2 \mathrm{~m} / \mathrm{s}\end{array}$ \\
\hline $\begin{array}{c}\text { Mantenimiento de canaletas, cajas de } \\
\text { aguas } \\
\text { lluvia y mobiliario urbano } \\
\end{array}$ & $\begin{array}{c}\text { Mantenimiento con el fin de eliminar obstrucciones } \\
\text { que puedan generar fallas en el drenaje de los } \\
\text { cuerpos de agua. }\end{array}$ & $\begin{array}{c}0,5 \mathrm{~m}>\text { Profundidad }(\mathrm{H})<1,0 \mathrm{~m} \\
\text { Velocidad de Flujo }(\mathrm{Vf})<2 \mathrm{~m} / \mathrm{s}\end{array}$ \\
\hline Modelación Hidrológica & $\begin{array}{l}\text { Para la actualización de las áreas de inundación se } \\
\text { generan hidrogramas con diferentes periodos de } \\
\text { retorno calibrados utilizando diferentes parámetros } \\
\text { de perdida y transformación provenientes de } \\
\text { estudios realizados previamente en el cuerpo de } \\
\text { agua. }\end{array}$ & $\begin{array}{l}0,5 \mathrm{~m}>\text { Profundidad }(\mathrm{H})<1,0 \mathrm{~m} \\
\text { Velocidad de Flujo }(\mathrm{Vf})<2 \mathrm{~m} / \mathrm{s}\end{array}$ \\
\hline Monitoreo de lluvias & $\begin{array}{l}\text { Se realiza seguimiento de las lluvias teniendo en } \\
\text { cuenta umbrales establecidos a los } 7 \text { días con el fin } \\
\text { de establecer puntos críticos en las localidades. }\end{array}$ & $\begin{array}{l}0,5 \mathrm{~m}>\text { Profundidad }(\mathrm{H})<1,0 \mathrm{~m} \\
\text { Velocidad de Flujo }(\mathrm{Vf})<2 \mathrm{~m} / \mathrm{s}\end{array}$ \\
\hline $\begin{array}{c}\text { Monitoreo del afluente y del caudal } \\
\text { del río }\end{array}$ & $\begin{array}{l}\text { Las estaciones ubicadas a lo largo del afluente } \\
\text { monitorean en tiempo real el movimiento de la } \\
\text { corriente y el caudal del río. }\end{array}$ & $\begin{array}{c}0,5 \mathrm{~m}>\text { Profundidad }(\mathrm{H})<1,0 \mathrm{~m} \\
\text { Velocidad de Flujo }(\mathrm{Vf})<2 \mathrm{~m} / \mathrm{s}\end{array}$ \\
\hline $\begin{array}{c}\text { Señalización de emergencia } \\
\text { preventiva }\end{array}$ & $\begin{array}{l}\text { Redes comunitarias que alerten el riesgo con el fin } \\
\text { de resguardarse. }\end{array}$ & $\begin{array}{l}0,5 \mathrm{~m}>\text { Profundidad }(\mathrm{H})<1,0 \mathrm{~m} \\
\text { Velocidad de Flujo }(\mathrm{Vf})<2 \mathrm{~m} / \mathrm{s}\end{array}$ \\
\hline
\end{tabular}

Fuente: Autores 


\section{CONCLUSIONES}

Se realizó el diagnostico en la Unidad de Planeamiento Zonal Tibabuyes frente al fenómeno de inundación, a partir de este fue posible establecer que la vulnerabilidad de la zona de estudio se debe principalmente a la cercanía de esta con el Humedal Juan Amarillo, el Humedal de la Conejera y el río Bogotá; así mismo, la principal consecuencia del riesgo existente se debe a la conformación de asentamientos informales y rellenos sobre los humedales, anudado al crecimiento desmedido en la UPZ y la ocupación del suelo con vocación agrícola, generando problemas de escorrentía e infiltración causante de los encharcamientos presentes en la UPZ Tibabuyes.

Para lograr resultados exitosos y definitivos, se debe tener como base, la Resolución 0631 de 2015, la cual "establece los parámetros y los valores límites máximo permisibles en los vertimientos puntuales a cuerpos de aguas superficiales y a los sistemas de alcantarillado público", y permitiría mejorar los índices de calidad en las aguas residuales tanto domésticas como industriales en la zona, pero no se hacer un seguimiento en su cumplimento de manera exhaustiva.

El aumento poblacional de la zona, relacionado con la informalidad tanto en las viviendas como las actividades económicas, hace que se presenten efectos negativos en el ecosistema de la ronda del río Bogotá, debido a la mala disposición de residuos sólidos, y la contaminación del agua, lo que, además, afecta la salud humana.

Se evaluaron las estrategias de mitigación del riesgo adaptadas con la información establecida por el IDIGER a través de la percepción de la población. De acuerdo con el análisis estadístico efectuado, se estableció que más del $75 \%$ de la muestra encuestada no posee conocimientos respecto a las estrategias para la mitigación del fenómeno de inundación; de igual manera, las medidas de tipo no estructural se encuentran rezagadas en la zona puesto que no se ha realizado su implementación.

Dentro de las medidas de mitigación evaluadas en la zona de estudio, a nivel general, se presentan falencias relacionadas con la falta de mantenimiento de las estructuras artificiales del río, la falta de inversión por parte del gobierno para el desarrollo de alertas a nivel local, generación de políticas públicas, mantenimiento de los cuerpos de agua e implementación de estructuras urbanas como forma de mitigar el riesgo y mejorar las condiciones de vida de los habitantes de la zona de estudio.

Se presentó un Plan de Acción de acuerdo con los escenarios de riesgo en la UPZ en donde las estrategias evaluadas están encaminadas a sensibilizar a la población respecto al riesgo inminente de inundación, mejorar el tipo de estructuras para el control de inundaciones e implementar estrategias inexistentes en las zonas, que servirán para preparar a la población ante una emergencia y reducir las condiciones de riesgo actuales.

Para implementar de un Plan de Acción en la zona de estudio es necesaria la percepción de la población y la intervención de las instituciones y actores de carácter público privado, que contribuyan en la prevención y gestión del riesgo, y en el fortalecimiento del cumplimento de la normativa vigente o, de ser el caso, su actualización; como sucede con la Norma Técnica Colombiana GTC 104, que establece Gestión del Riesgo Ambiental y data de año 2009

Se debe trabajar articuladamente en el Plan de Ordenamiento Territorial con el Plan de Ordenación y Manejo de la Cuenca Hidrográfica del río Bogotá, establecido en la Resolución 0957 
de 2019, revisando las estrategias complementarias o faltantes en la gestión del riesgo de inundación y los asociados a esta.

\section{CONTRIBUCIÓN DE LA AUTORÍA}

Primer autor: metodología de participación, conceptualización, escritura - borrador original. Segundo autor: investigación, conceptualización. Tercer autor: administración del proyecto, logística, análisis de datos y modelación de la encuesta, revisión y edición, escritura - borrador original Cuarto autor: revisión y edición.

\section{AGRADECIMIENTOS}

En primer lugar, deseamos agradecer al PhD(c). Didier Camilo Sierra por la dedicación y apoyo que ha brindado a este trabajo, por el respeto a las sugerencias e ideas y por la dirección del articulo y del trabajo de tesis elaborado por Ayala, A, \& Valbuena, T. (2019), por parte de la Universidad Sergio Arboleda. Propuesta conceptual para la mitigación de riesgo de inundación en la Unidad de Planeamiento Zonal (UPZ) Tibabuyes, Iocalidad de Suba, el cual fue la base para el desarrollo del artículo.

\section{LITERATURA CITADA}

Alcaldía Local de Suba. (2012). PLAN AMBIENTAL LOCAL SUBA 2013-2016. Bogotá: Secretaría Distrital de Ambiente. Recuperado de http://www.ambientebogota.gov.co/documents/10157/2883165/ PAL+Suba+2013-2016.pdf

Alkire, S., \& Foster, J. (2011). Understandings and Misunderstandings of Multidimensional Poverty Measurement. OPHI Working Papers, 43. Oxfordshire: University of Oxford. Recuperado de https://www.ophi.org.uk/wp-content/ uploads/ophi-wp43.pdf

Alkire, S., \& Santos, M. E. (2010). Acute Multidimensional Poverty: A New Index for Developing Countries. OPHI Working Papers, 38. Oxfordshire: University of Oxford. Recuperado de https://www.ophi.org.uk/wp-content/uploads/ OPHI-wp38_with_note.pdf
Angulo, R. C., \& Diaz, Y. (2011). Índice de Pobreza Multidimensional para Colombia. Bogotá: Departamento Nacional de Planeación. Recuperado de https://colaboracion.dnp.gov.co/cdt/estudios\%20econmicos/382.pdf

Corporación Autónoma Regional (CAR). (2019). Resolución 957 de 2019 "Por la cual se aprueba el ajuste y actualización del POMCA río Bogotá". Corporación Autónoma Regional. Recuperado de https://www.car.gov.co/vercontenido/3691

Gobernación de Cundinamarca. (2017). Estudio de Crecimiento y Evolución de la Huella Urbana para los Municipios que conforman el área Bogotá Región. Bogotá: Secretaría Distrital de Planeación. Recuperado de http://www.sdp.gov.co/sites/default/files/diagnostico_de_la_huella_urbana_de_ bogota_y_20_municipios_de_1997_a_2016.pdf

Guzmán, T. (2015). Procesos de expansión urbana en la ciudad colombiana. Efectos de la transformación del municipio de Chía (2002-2012) (estudio de caso). Universidad Colegio Mayor de Nuestra Señora del Rosario. Bogotá. Recuperado de http://repository.urosario.edu.co/bitstream/ handle/10336/11687/1.019.034.788\%20-\%20 2015. pdf? sequence $=1$

Hernández, D., Serrano, R. (2012). Propuesta de mitigación para los sectores críticos de inundación en la localidad de Suba, Bogotá D.C. Teoría Y Praxis Investigativa, 8(1), 8 - 20. Recuperado de http://revia.areandina.edu.co/ojs/index.php/ $\mathrm{Pp} /$ article/view/397/429

Instituto de Estudios Urbanos. (2011). Diagnóstico Local, Localidad de Suba. Recuperado de http:// www.institutodeestudiosurbanos.info/dmdocuments/cendocieu/coleccion_digital/Conformacion_Vereda_Chorrillos/Diagnostico_Suba-Hospital_Suba-2011.pdf

Instituto Distrital de Gestión de Riesgos y Cambio Climático (IDIGER). (2018). Documento técnico de soporte: Estudios básicos «Amenazas por inundaciones en perspectiva de cambio climático». Bogotá: Secretaría Distrital de Planeación. Recuperado de http://www.sdp.gov.co/sites/ default/files/4-DOCUMENTO-TECNICO-DE-SOPORTE/Gestion\%20del\%20Riesgo.\%20Amenazas\%20inundacion\%20Urbano.pdf

Instituto Distrital de Gestión de Riesgos y Cambio Climático (IDIGER). (s. f.). Caracterización General del Escenario de Riesgo por Inundación. Bogotá: Instituto Distrital de Gestión de Riesgos 
y Cambio Climático. Recuperado de https://www. idiger.gov.co/rinundacion

Instituto Geográfico Agustín Codazzi (2019). Aerografía de la Localidad de Suba año 1995 y año 2004.[Figura 3]. Recuperado de https://geoportal.igac.gov.co/contenido/consulta

Rodríguez, M, y Guarín, R. (2015). ANÁLISIS DE LOS PROGRAMAS DE PREVENCIÓN Y MITIGACIÓN DE LA GESTIÓN DEL RIESGO EN LA LOCALIDAD DE SUBA - BOGOTÁ. (Tesis de especialización). Universidad Santo Tomas, Bogotá, Colombia. Recuperado de https://repository.usta.edu.co/ handle/11634/2246?show=full

Serrano, R., \& Romero, D. (2017). Propuesta de mitigación para los sectores críticos de inundación

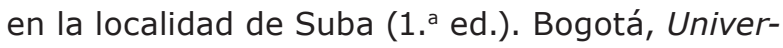
sidad Piloto de Colombia. Recuperado de https:// books.google.com.co/books?id=4ptYDwAAQBAJ $\& p g=P T 21 \& l p g=P T 21 \& d q=$ riesgo $\# v=$ onepage $\& q$ $=$ riesgo $\& \mathrm{f}=$ false

Secretaría Distrital de Planeación (SDP). (2014). Proyecciones de población por localidades para Bogotá 2016-2020. Bogotá: Secretaría Distrital de Planeación. Recuperado de http://www.sdp. gov.co/sites/default/files/boletin69.pdf

Secretaria Distrital de Planeación. (2017). Localidad de Suba Diagnóstico de los principales aspectos territoriales, de infraestructura, demográficos y socioeconómicos. Bogotá: Secretaría Distrital de Planeación. Recuperado de http://www.sdp.gov. co/gestion-estudios-estrategicos/informacioncartografia-y-estadistica/repositorio-estadistico/ monografia-localidad-de-suba-2017\%5D

Unidad Nacional para la Gestión del Riesgo de Desastres. (2018). Atlas de riesgo de Colombia: revelando los desastres latentes. Bogotá: Unidad Nacional para la Gestión del Riesgo de Desastres. Recuperado de https://repositorio.gestiondelriesgo.gov.co/handle/20.500.11762/27179

Vallejo, A, y Vélez, J. (2009). La percepción del riesgo en los procesos de urbanización del territorio. Letras Verdes Revista Latinoamericana de Estudios Socioambientales. Recuperado de https:// revistas.flacsoandes.edu.ec/letrasverdes/article/view/833
Conflicto de Intereses Los autores declaran no tener ningún conflicto de intereses 